\title{
The importance of endothelial protection: the emerging role of defibrotide in reversing endothelial injury and its sequelae
}

\author{
Paul G. Richardson (iD) ${ }^{1 凶}$, Marta Palomo ${ }^{2,3}$, Nancy A. Kernan ${ }^{4}$, Gerhard C. Hildebrandt (iD) ${ }^{5}$, Nelson Chao (D) ${ }^{6}$ and Enric Carreras ${ }^{2}$
}

(c) The Author(s) 2021

Hepatic veno-occlusive disease/sinusoidal obstruction syndrome (VOD/SOS), a potentially life-threatening complication of hematopoietic cell transplantation $(\mathrm{HCT})$, results from prolonged sinusoidal endothelial cell activation and profound endothelial cell damage, with sequelae. Defibrotide, the only drug approved in the United States and Europe for treating VOD/SOS post-HCT, has European Commission orphan drug designation for preventing graft-versus-host disease (GvHD), associated with endothelial dysfunction. This endothelial cell protector and stabilizing agent restores thrombo-fibrinolytic balance and preserves endothelial homeostasis through antithrombotic, fibrinolytic, anti-inflammatory, anti-oxidative, and anti-adhesive activity. Defibrotide also preserves endothelial cell structure by inhibiting heparanase activity. Evidence suggests that downregulating p38 mitogen-activated protein kinase (MAPK) and histone deacetylases (HDACs) is key to defibrotide's endothelial protective effects; phosphatidylinositol 3-kinase/Akt (PI3K/AKT) potentially links defibrotide interaction with the endothelial cell membrane and downstream effects. Despite defibrotide's being most extensively studied in VOD/SOS, emerging preclinical and clinical data support defibrotide for treating or preventing other conditions driven by endothelial cell activation, dysfunction, and/or damage, such as GvHD, transplant-associated thrombotic microangiopathy, or chimeric antigen receptor T-cell (CAR-T) therapy-associated neurotoxicity, underpinned by cytokine release syndrome and endotheliitis. Further preclinical and clinical studies will explore defibrotide's potential utility in a broader range of disorders resulting from endothelial cell activation and dysfunction.

Bone Marrow Transplantation (2021) 56:2889-2896; https://doi.org/10.1038/s41409-021-01383-x

\section{INTRODUCTION}

Defibrotide, a polydisperse mixture of predominantly singlestranded polydeoxyribonucleotide sodium salts, was identified in the 1960s in the search for potential treatments for coagulation and thrombotic disorders that would have a lower risk of hemorrhage than other anticoagulants. Originally derived from bovine lung, defibrotide is now produced via controlled depolymerization of porcine intestinal DNA [1-3]. Defibrotide has demonstrated antithrombotic, fibrinolytic, anti-inflammatory, anti-oxidative, and anti-adhesive activity, but has shown no systemic anticoagulant effects, with no effects on coagulation parameters such as partial thromboplastin or prothrombin times $[1,4]$.

Defibrotide was first approved for clinical use in Italy in 1986 to treat deep vein thrombosis, an indication expanded in 1993 to include treatment and prevention of vascular disease with risk of thrombosis [5]. By the mid-1990s, defibrotide was considered for treating severe veno-occlusive disease/sinusoidal obstruction syndrome (VOD/SOS) as part of a study searching for potential novel therapies targeting endothelial injury; its positive impact on endothelial stress was confirmed with correlative studies in the same setting $[6,7]$. As of 2020 , defibrotide is the only drug approved for treating hepatic VOD/SOS. In the United States, defibrotide is indicated for treatment of adult or pediatric patients with hepatic VOD/SOS with renal or pulmonary dysfunction following hematopoietic cell transplantation (HCT) [2]. In Europe, defibrotide is indicated for treatment of severe hepatic VOD/SOS following HCT in patients aged $>1$ month [8]. In addition, the European Commission granted orphan drug designation to defibrotide in 2013 for prevention of graft-versus-host disease (GvHD) [9].

VOD/SOS, a potentially life-threatening HCT complication, is caused by prolonged sinusoidal endothelial cell activation and subsequent injury from radiation or toxic metabolites of chemotherapy within HCT conditioning regimens [10-12]. Endothelial cell damage increases expression of von Willebrand factor (vWF), which stimulates platelet aggregation, and tissue factor, which promotes activation of coagulation factors. Endothelial cell damage also increases expression of inflammatory mediators, such as intercellular adhesion molecule-1 (ICAM-1) and tumor necrosis factor- $a$ (TNF-a), and releases heparanase, which results in loss of cytoskeletal structure [13-16]. In the liver, as the sinusoidal endothelium deteriorates, gaps form in the endothelial lining, allowing cellular debris to enter the space of

\footnotetext{
'Jerome Lipper Multiple Myeloma Center, Dana-Farber Cancer Institute, Harvard Medical School, Boston, MA, USA. ${ }^{2}$ Barcelona Endothelium Team, Josep Carreras Leukaemia Research Institute, Hospital Clinic/University of Barcelona Campus, Barcelona, Spain. ${ }^{3}$ Hematopathology, Department of Pathology, Centre de Diagnostic Biomedic (CDB), Hospital Clinic de Barcelona, Institut d'Investigacions Biomediques August Pi i Sunyer (IDIBAPS), Universitat de Barcelona, Barcelona, Spain. ${ }^{4}$ Pediatric BMT Service, Memorial Sloan Kettering Cancer Center, New York, NY, USA. ${ }^{5}$ Markey Cancer Center, University of Kentucky, Lexington, KY, USA. ${ }^{6}$ Duke Cancer Institute, Duke University Medical Center, Durham, NC, USA. ${ }^{凶}$ email: Paul_Richardson@dfci.harvard.edu
} 


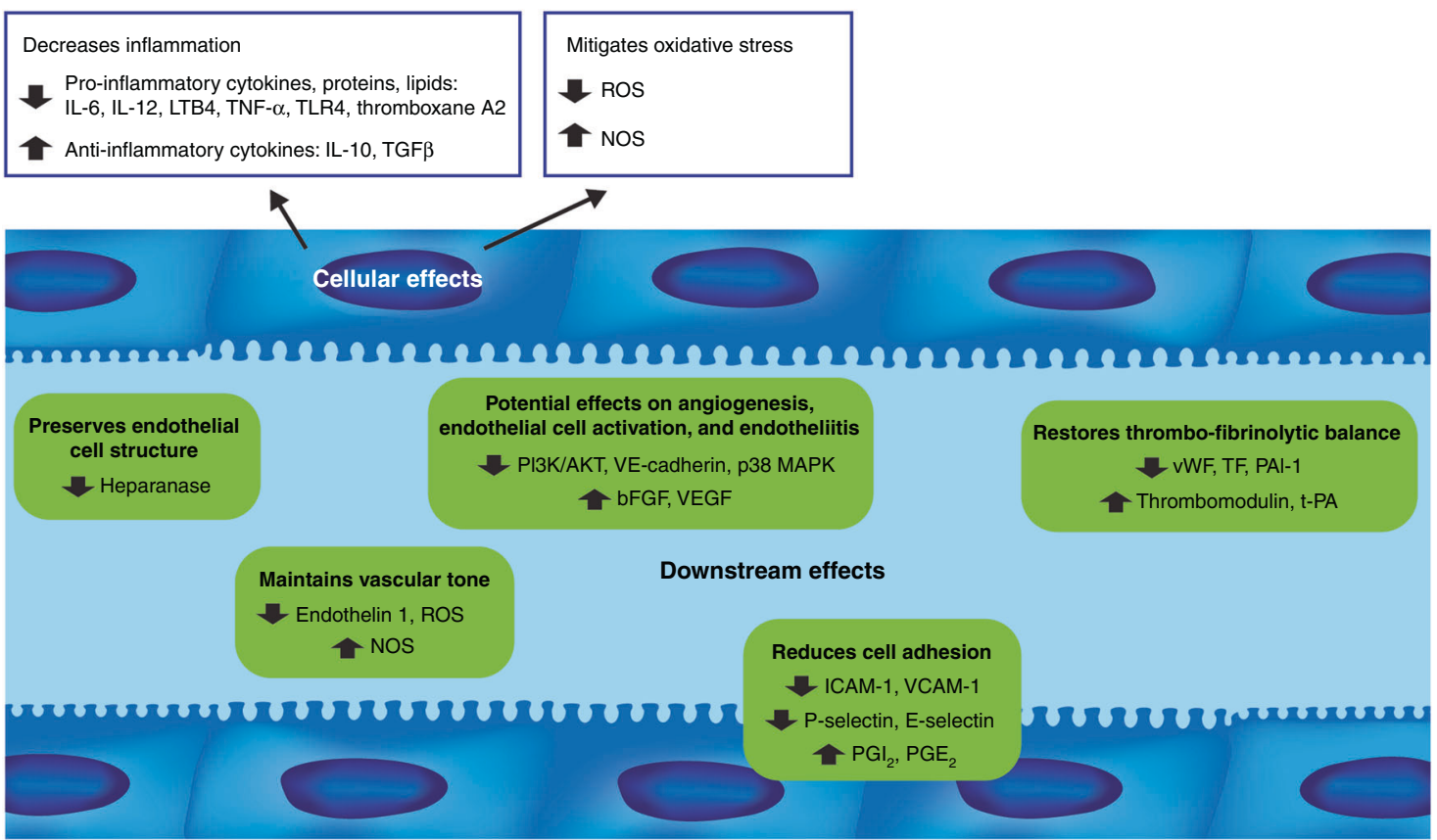

Fig. 1 Proposed cellular and downstream effects of defibrotide. IL interleukin, LTB4 leukotriene B4, TNF- $\alpha$ tumor necrosis factor- $\alpha$, TLR4 Tolllike receptor 4, TGF $\beta$ transforming growth factor- $\beta$, ROS reactive oxygen species, NOS nitric oxide synthase, PI3K/AKT phosphatidylinositol 3-kinase/Akt, VE-cadherin vascular endothelial cadherin, MAPK mitogen-activated protein kinase, bFGF basic fibroblast growth factor, VEGF vascular endothelial growth factor, vWF von Willebrand factor, TF tissue factor, PAI-1 plasminogen activator inhibitor-1, t-PA tissue plasminogen activator, ICAM-1 intercellular adhesion molecule, VCAM-1 vascular cell adhesion molecule-1, PGI ${ }_{2}$ prostaglandin I2, $^{2}$ $\mathrm{PGE}_{2}$ prostaglandin $\mathrm{E} 2$.

Disse, leading to sinusoidal narrowing. This is accompanied by downstream embolism of sinusoidal endothelial cells which cause a progressive blockage of sinusoidal flow [1, 11, 15, 17]. Changes induced by endothelial cell activation induce a shift in phenotype, from antithrombotic to procoagulant, with hypofibrinolysis reflected by downregulation of tissue plasminogen activator and markedly increased plasminogen activator inhibitor-1 (PAl-1) levels [18], leukocyte adhesion molecule expression, cytokine production, and loss of vascular integrity. These changes appear to be a common pathogenic mechanism underlying a range of conditions [19]. Notably, endothelial cells are not all the same, performing different functions depending on their location in the body. The endothelium arises from cell differentiation in the mesoderm; however, other cell lineages may also transdifferentiate into endothelial cells. Therefore, features such as Weibel-Palade bodies or fenestrae are not found in every endothelial cell. Consequently, endothelial cell heterogeneity has posed a challenge for developing treatments [20].

This article discusses and reviews the proposed mechanism of action of defibrotide, an endothelial cell protector.

\section{Pharmacological characteristics of defibrotide}

Defibrotide is a mixture of $90 \%$ single-stranded phosphodiester oligonucleotides and 10\% double-stranded phosphodiester oligonucleotides. Within this mixture, various aptamers have been identified with biological actions in vitro, including thrombin inhibition and cathepsin $G$ inhibition $[3,21]$. The polyanionic nature of the single-stranded DNA aptamers interferes with cationic proteins that cause vascular instability [22]. These single-stranded DNA aptamers then modulate the function of cationic proteins secreted by activated neutrophils in the context of neutrophil extracellular traps [22, 23]. However, defibrotide degrades to several different products in vivo, so the identity of the clinically active derivative is unclear [4].
A lack of immunogenicity was demonstrated in animal models after prolonged defibrotide administration. A preclinical study using rats and dogs found that treatment with varying doses of defibrotide for a 3-month period did not result in the production of anti-defibrotide, anti-heparin platelet factor 4 (PF4) or antiphospholipid antibodies. This is a notable observation as it contrasts with polyelectrolytes such as heparin which are capable of interacting with endogenous proteins, leading to the formation of antibodies such as anti-heparin PF4 [24].

Defibrotide has a relatively short half-life. In pharmacokinetic studies of healthy volunteers, the mean (standard deviation) halflife was $0.71(0.35) \mathrm{h}$ after one $6.25-\mathrm{mg} / \mathrm{kg}$ dose of defibrotide [8]. A Japanese study reported a mean (standard deviation) half-life of $0.47(0.10) \mathrm{h}$ after defibrotide $6.25 \mathrm{mg} / \mathrm{kg}$ administration to healthy volunteers as a 2 -h infusion [25]. Given this relatively short half-life, defibrotide must be administered every $6 \mathrm{~h}$; it can also clear rapidly in case of adverse events [8].

\section{Cellular effects of defibrotide}

Defibrotide protects endothelial cells via modulation of a wide, complex range of molecular pathways (Fig. 1 and Table 1). In a human endothelial cell line, defibrotide attached to the external cell membrane and then became internalized via macropinocytosis in a concentration-, temperature-, and time-dependent fashion [26]. However, the interaction between defibrotide and the endothelial cell membrane, without internalization, was sufficient to induce anti-inflammatory and antioxidant responses [26]. Defibrotide anti-inflammatory effects include reduced release of inflammatory mediators such as interleukin 6 (IL-6), thromboxane $A 2$, leukotriene B4, and TNF [3], while antioxidant effects include attenuation of reactive oxygen species (ROS) generation and restoration of endothelial nitric oxide synthase levels during oxidative stress in vitro [26]. Interaction with adenosine receptors on the endothelial cell membrane had been considered a critical 
Table 1. Proposed cellular effects of defibrotide.

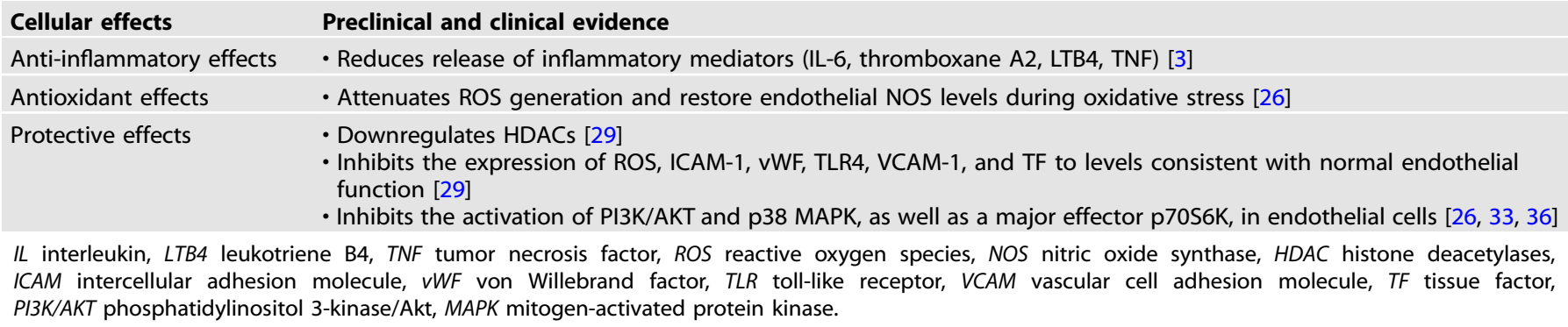

step in defibrotide's mechanism of action [27]. In a study in murine dendritic cells, defibrotide-induced production of interleukin 10 was completely inhibited by incubation with the adenosine receptor antagonist 8-p-sulfophenyltheophylline, suggesting that defibrotide acted as an agonist at the adenosine receptor [28]. However, in human endothelial cell line experiments, blocking adenosine receptors with 8-p-sulfophenyltheophylline had no effect on defibrotide attaching to cells and no specific receptor for defibrotide was identified [26].

Histone deacetylase (HDAC) downregulation is thought to be a key mechanism of defibrotide's endothelial protective effects [29]. HDACs are important epigenetic factors regulating proinflammatory gene expression in tissues $[30,31]$. In endothelial cells, HDACs appear to be involved in modulating inflammation and proliferation in response to shear stresses [30]. Western blot and immunofluorescence techniques showed that defibrotide normalized overexpression of HDAC1 in a dose-dependent manner in human umbilical cord vein endothelial cells exposed to sera of patients with chronic kidney disease, a condition associated with endothelial dysfunction [29]. Furthermore, in endothelial cells exposed to chronic kidney disease patients' sera, defibrotide inhibited expression of ROS, ICAM-1, vWF, and Toll-like receptor 4 to levels consistent with normal endothelial function [29]. Previous studies showed that HDAC expression is regulated by the phosphatidylinositol 3-kinase/Akt (PI3K/AKT) pathway, as demonstrated by a reduction in shear-stress-induced expression of HDACs by PI3K inhibitors [30, 32]. Several early HCT-associated complications seem to have a more microvascular (i.e., small blood vessel) than macrovascular (i.e., large blood vessel) location.

Defibrotide has inhibited PI3K/AKT activation in endothelial cells derived from human umbilical vein and an immortalized human microvascular cell line induced by HCT patients' sera [33] and in microvascular endothelial cells with AKT activation stimulated by exposure to GvHD patient serum [34]. In addition, defibrotide blocked activation of p70S6K, a major PI3K/AKT pathway effector, in microvascular endothelial cells [35]. Furthermore, defibrotide prevented increased HDAC1 and HDAC2 expression in endothelial cell cultures incubated with P740-Y-P, a PI3K/AKT pathway activator [29]. Thus, it appears that one mechanism of defibrotide's endothelial protective effects reduced HDAC upregulation through PI3K/AKT pathway inhibition, making PI3K/AKT the potential link between defibrotide's interaction with the endothelial cell membrane and its downstream effects. Moreover, defibrotide has decreased activation of p38 mitogen-activated protein kinase (MAPK), which occurs even in the context of micropinocytosis inhibition. This suggests that defibrotide may act at the endothelial cell membrane to alter intracellular p38 MAPK signaling [26]. Supporting this as a potential key construct to defibrotide's mechanism of action, p38 MAPK pathway upregulation activates pro-inflammatory cytokines, such as IL-6, TNF-a, and $\mathrm{IL}-1 \beta$, thus contributing to numerous inflammatory pathologies, including endotheliitis [36]. In summary, p38 MAPK pathway downregulation may be an important additional mechanism of defibrotide's anti-inflammatory and endothelial protective cellular effects.

\section{Downstream effects of defibrotide}

Defibrotide acts on multiple pathways that affect endothelial homeostasis (Fig. 1 and Table 2). Defibrotide protects endothelial cells and reduces their activation and subsequent dysfunction via antithrombotic, fibrinolytic, anti-inflammatory, anti-oxidative, antiadhesive, anti-apoptotic, and anti-angiogenic mechanisms, thereby restoring thrombotic-fibrinolytic balance and preserving endothelial homeostasis $[1,3,4,33,37-41]$.

Effects on thrombo-fibrinolytic balance. Several in vitro studies have demonstrated that defibrotide modulates antithrombotic and fibrinolytic factors. Three aptamers from defibrotide were shown to be potent inhibitors of thrombin-induced platelet aggregation, thromboxane biosynthesis, and fibrin clot formation in vitro [21]. In human umbilical vein endothelial cells (macrovascular) and microvascular endothelial cells, defibrotide prevented increased expression of VWF induced by exposure to sera from autologous HCT recipients or newly diagnosed GvHD patients [33, 34]. Defibrotide also reduced the increased endothelial cell matrix reactivity toward platelets induced by GvHD serum exposure [34]. In microvascular endothelial cells, defibrotide reduced lipopolysaccharide-induced tissue factor expression, tissue factor procoagulant activity, and tissue factor antigen [40]; in macrovascular cells, incubation with defibrotide stimulated thrombomodulin expression [42].

Fibrinolytic properties were demonstrated in an in vitro experiment in which defibrotide bound to human plasmin and enhanced plasmin activity to degrade fibrin clots formed by fibrinogen, plasminogen, and thrombin [39]. In macro- and microvascular endothelial cells, defibrotide prevented lipopolysaccharide-induced increase in PAI-1 expression and enhanced lipopolysaccharideinduced increase in tissue plasminogen activator antigen expression, resulting in a net increase in fibrinolytic activation with defibrotide [40]. Human study findings mirror these in vitro results. In healthy volunteers, a single defibrotide dose counteracted a natural circadian fall in tissue plasminogen activator and accelerated the PAl-1 level decrease seen in placebo-treated participants, suggesting defibrotide acts to stimulate circulating fibrinolytic activity [38]. In another healthy volunteer study, a single defibrotide dose induced an increased level of systemic tissue factor pathway inhibitor, which plateaued between 5 and $20 \mathrm{~min}$ post-dose and returned to basal levels by $40 \mathrm{~min}$ post-dose, a profile similar to that following a single dose of low-molecular weight heparins, enoxaparin, and nadroparin [37]. In patients with VOD/SOS, defibrotide decreased PAI- 1 and increased tissue plasminogen activator levels, increasing overall fibrinolytic activity [43].

Although defibrotide has been shown to reduce platelet adhesion and aggregation, it has not shown significant systemic anticoagulant effects or effects on coagulation parameters, such as partial and activated thromboplastin times and prothrombin time [4, 44]. An 
Table 2. Proposed downstream effects of defibrotide.

\section{Downstream effects}

Effects on thrombo-fibrinolytic balance

Reduction of cell adhesion

Anti-inflammatory/antioxidant properties

Endothelial cell protection and anti-apoptotic effects

Anti-angiogenic effects

\section{Preclinical and clinical evidence}

- Inhibits thrombin-induced platelet aggregation, thromboxane biosynthesis, and fibrin clot formation [21]

- Prevents increased expression of vWF $[33,34]$

- Reduces reactivity of the endothelial cell matrix toward platelets induced by exposure to GvHD serum [34]

- Reduces LPS-induced tissue factor expression, tissue factor procoagulant activity, and tissue factor antigen in microvascular endothelial cells [40]

- Stimulates thrombomodulin expression in macrovascular endothelial cells [42]

- Enhances the activity of plasmin to degrade fibrin clots formed by fibrinogen, plasminogen, and thrombin [39]

- Prevents LPS-induced increase in PAI-1 expression and enhanced LPS-induced increase in t-PA antigen expression, resulting in a net increase in fibrinolytic activation [40]

- Inhibits leukocyte adhesion by interfering with LFA-1/ICAM-mediated leukocyte transmigration in vitro [45] and by suppressing P-selectin in rats [46]

- Stimulates the production of $\mathrm{PGI}_{2}$ and $\mathrm{PGE}_{2}$ [38]

- Suppresses acute GvHD serum-induced expression of VCAM-1, ICAM-1, and vascular-endothelial cadherin [34]

- Inhibits mononuclear cells and CD3+ T cells while downregulating expression of the adhesion molecules E-selectin, P-selectin, VCAM-1, and ICAM-1 [48]

- Blocks the increased expression of ICAM-1 in patients undergoing autologous HCT [33]

- Decreases the release of inflammatory mediators (IL-6, thromboxane A2, LTB4, TNF, and ROS) $[3,26]$

- Decreases levels of the pro-inflammatory cytokines (IFN $\gamma$, TNF- $\alpha$, IL-6, and IL-12) [48]

- Increases levels of the anti-inflammatory mediators (TGF $\beta$ and IL-10) [48]

- Maintains vascular tone by antagonizing the vasoconstrictor activity of endothelin 1 and enhancing the production of NO and NOS [3]

- Protects endothelial and epithelial cells from F-Ara-induced apoptosis [41]

- Inhibit the activity and cell surface expression of heparanase, VEGF, ICAM-1, and E-selectin in multiple myeloma and mesothelioma cells [49]

- Binds and mobilizes bFGF, while protecting it from oxidative and protease degradation and potentiating its binding to FGFR1-IIlc [53]

- Inhibits formation of new blood vessels and reduces tumor microvascular density [35]

- Reduces acute GvHD-induced expression of VE-cadherin and suppresses endothelial cell proliferation and endothelial cell tube formation [34]

$\checkmark W F$ von Willebrand factor, HCT hematopoietic cell transplantation, GvHD graft-versus-host disease, LPS lipopolysaccharide, PAl-1 plasminogen activator inhibitor-1, $t-P A$ tissue plasminogen activator, $L F A-1$ lymphocyte function-associated antigen-1, ICAM intercellular adhesion molecule, $P G I_{2}$ prostaglandin 12 , $P G E_{2}$ prostaglandin E2, VCAM vascular cell adhesion molecule, IL interleukin, LTB4 leukotriene B4, TNF tumor necrosis factor, ROS reactive oxygen species, IFN $\gamma$ interferon- $\gamma$, TGF $\beta$ transforming growth factor- $\beta$, NO nitric oxide, NOS nitric oxide synthase, VEGF vascular endothelial growth factor, $b F G F$ basic fibroblast growth factor, FGFR fibroblast growth factor receptor.

in vitro study using human blood products showed that defibrotide increased plasmin activity with no direct effect on plasminogen activation to plasmin, suggesting a local fibrinolytic potential for defibrotide [39].

Reduction of cell adhesion. Defibrotide has demonstrated antiadhesive properties in vitro and in preclinical and human studies. In cell cultures, defibrotide inhibited leukocyte adhesion to endothelial cells under basal conditions and after endothelial cell stimulation. Defibrotide inhibited leukocyte adhesion by interfering with lymphocyte function-associated antigen-1/intercellular adhesion molecule-mediated leukocyte transmigration in vitro [45] and by suppressing P-selectin in rats [46]. In platelet-perfused guinea pig hearts, defibrotide administration resulted in selective stimulation of prostaglandin 12 but had no effect on release of prostaglandin E2 or thromboxane [47]. However, in human volunteers, defibrotide stimulated production of prostaglandin 12 and prostaglandin E2, likely through stimulating the arachidonate pathway in leukocytes or by platelet-leukocyte interactions [38].

In vitro studies also examined defibrotide's effects on GvHD patient serum-induced changes in adhesion parameters. In microvascular endothelial cell cultures, defibrotide significantly suppressed acute GvHD serum-induced expression of vascular cell adhesion molecule-1, ICAM-1, and vascular-endothelial cadherin [34]. In macrovascular endothelial cell cultures exposed to GvHD sera followed by defibrotide, trans-endothelial migration of mononuclear cells and CD3 $+\mathrm{T}$ cells was inhibited, along with a downregulated expression of adhesion molecules E-selectin, P-selectin, vascular cell adhesion molecule-1, and ICAM-1 compared with cultures not treated with defibrotide [48]. Results were similar in macrovascular and microvascular endothelial cells exposed to sera from autologous HCT patients, in which defibrotide blocked increased expression of ICAM-1 [33]. These findings suggest that defibrotide's protective actions in the endothelium include downregulating expression of endothelial adhesion molecules overexpressed in response to GvHD or HCT.

Anti-inflammatory and antioxidant properties. In endothelial cells, defibrotide exerts anti-inflammatory and antioxidant properties by decreasing release of inflammatory mediators such as IL-6, thromboxane $A 2$, leukotriene $B 4, T N F$, and $\operatorname{ROS}[3,26]$. In a mouse model of GvHD, mice with ongoing acute GvHD post-HCT had increased levels of pro-inflammatory cytokines interferon- $\gamma$, TNF-a, $\mathrm{IL}-6$, and interleukin 12, with reduced levels of anti-inflammatory mediators transforming growth factor- $\beta$ and interleukin 10, compared with control mice. Prophylactic defibrotide treatment produced a marked decrease in pro-inflammatory mediators and an increase in anti-inflammatory mediators compared with untreated mice [48]. Defibrotide also maintained vascular tone by antagonizing endothelin 1 vasoconstrictor activity and enhancing production of nitric oxide and nitric oxide synthase. These effects are potentiated by reduction of ROS formation, which in 
turn improves the bioavailability and effectiveness of endothelium-derived nitric oxide [3].

Endothelial cell protection and anti-apoptotic effects. The pathways through which defibrotide protects against chemotherapyrelated endothelial damage do not appear to impact chemotherapy-induced antitumor activity. In microvascular endothelial cell culture, apoptosis was induced by incubation with F-Ara, the active metabolite of fludarabine; defibrotide pretreatment or coadministration protected endothelial cells from F-Ara-induced apoptosis [41]. Similar defibrotide protective effects were observed for epithelial cells from keratinocyte and alveolar cell lines, a potentially relevant observation given that skin is the most frequent site of acute GvHD manifestation and the lung significantly contributes to transplant-related mortality in idiopathic pneumonia syndrome and diffuse alveolar hemorrhage [41]. Another study looked at in vitro effects of defibrotide combined with a series of antitumor agents, including chemotherapies and biologic agents, on multiple myeloma and endothelial cells [49]. Defibrotide had no effect on these agents' antitumor activity and no antitumor activity of its own. However, in the same study, defibrotide inhibited heparanase activity in multiple myeloma cells and, while adding exogenous heparanase increased the invasion potential of multiple myeloma cells, defibrotide treatment abolished this effect [49]. Furthermore, the interaction of multiple myeloma cells with microvascular endothelial cells increased expression of heparanase, vascular endothelial growth factor, ICAM-1, and E-selectin in endothelial cells; defibrotide suppressed these effects [49]. Another study also found that defibrotide inhibited heparanase activity and tumor growth in mesothelioma cells [50]. Heparan sulfate proteoglycans are crucial in maintaining the structure and stability of the extracellular matrix and basement membrane [51], so inhibiting heparanase expression may contribute to defibrotide's protective effects on the endothelium. Interestingly, high levels of heparanase in recipients compared with donors have been associated with acute and chronic GvHD following HCT, suggesting a role for heparanase in hyperactivation of donor $T$ cells toward recipient tissues and a potential rationale for heparanase-targeting treatments [52].

Anti-angiogenic effects. Defibrotide effects on angiogenic mechanisms appear to be complex. In vitro, defibrotide has been shown to bind with high affinity to basic fibroblast growth factor (bFGF) and mobilize bFGF from its binding sites on the endothelial extracellular matrix, while protecting bFGF from oxidative and protease degradation and potentiating its binding to fibroblast growth factor receptor [53]. As bFGF is known to promote formation of microvessels and stimulate expression of vascular endothelial growth factor, these results suggest that defibrotide's action in VOD/SOS may be partly related to its ability to promote partial revascularization of injured hepatic tissue, through direct bFGF enhancement and indirect effects on vascular endothelial growth factor-induced angiogenesis [53]. However, evidence also suggests that defibrotide has antiangiogenic activity. As discussed previously, defibrotide inhibits $\mathrm{PISK} / \mathrm{AKT}$, an important signaling pathway for angiogenesis [35]. In vitro and in vivo data suggest that defibrotide anti-angiogenic effects occur, not by disrupting tumor endothelium, but by inhibiting formation of new blood vessels and reducing tumor microvascular density [35]. Similarly, in a mouse model of mesothelioma, defibrotide treatment inhibited tumor growth associated with impaired tumor vascular density [50]. In endothelial cell culture, defibrotide reduced acute GvHDinduced expression of vascular endothelial-cadherin, an adherence junction protein involved in angiogenesis and vascular integrity; defibrotide also suppressed endothelial cell proliferation and endothelial cell tube formation [34].

\section{Predictive biomarkers for defibrotide}

Identification of potential biomarkers for defibrotide efficacy has proven complex given its pleiotropic effects. Kaleelrahman et al. studied the potential role of PAI-1 levels in diagnosing hepatic VOD/SOS post-HCT and response to subsequent defibrotide treatment. In a study involving five patients with suspected VOD/SOS post-HCT, PAI-1 levels decreased after defibrotide treatment in the four patients showing VOD/SOS resolution, but remained elevated in the patient who did not respond to defibrotide [54]. In an exploratory analysis of a phase 2 dosefinding study of defibrotide in 151 patients with severe VOD/SOS post-HCT, PAl-1 plasma concentrations were lower (although this difference was not statistically significant) at Days 7 and 14 of defibrotide treatment compared with baseline in patients with complete response and in patients who survived beyond Day 100 [55]. Decreased or stable bilirubin has also been associated with better response to defibrotide [55], suggesting that early bilirubin and/or PAl-1 changes could be potential pharmacodynamic markers for efficacy, as could reduced serum creatinine based upon results of an earlier study, along with improved platelet count [7].

\section{Clinical implications}

Given its role in promoting endothelial homeostasis, defibrotide has therapeutic potential for conditions driven by endothelial dysfunction. Based on its identified pharmacologic actions, defibrotide has been evaluated in disorders involving endothelial cell activation, endotheliitis, and/or endothelial damage.

VOD/SOS. Thus far, defibrotide has been most extensively studied for treating and preventing VOD/SOS post-HCT. Defibrotide is currently the only drug approved for treatment of adult and pediatric patients with hepatic VOD/SOS following HCT $[2,8]$. In a phase 3 open-label, historically controlled study in 102 patients with VOD/SOS with multi-organ failure, Day 100 survival post-HCT was $38 \%$ with defibrotide versus $25 \%$ in the historical control group $(P=0.0109)$, with complete response rates of $26 \%$ versus $13 \%$, respectively $(P=0.0160)$ [56]. These findings were replicated in a larger open-label, expanded-access study of defibrotide in 1000 patients with VOD/SOS with or without multi-organ dysfunction following HCT (Day 100 survival: 59\%). Among patients with multi-organ dysfunction $(n=512)$, Day 100 survival was $50 \%$ [57]. Defibrotide also demonstrated efficacy for prevention of VOD/SOS in a phase 3, open-label, randomized trial of 356 high-risk pediatric patients undergoing $\mathrm{HCT}$; prophylactic defibrotide reduced VOD/SOS incidence at 30 days post-HCT versus controls ( $12 \%$ vs $20 \% ; P=0.0488$ ) [58]. An ongoing phase 3 trial of defibrotide for VOD/SOS prophylaxis (NCT02851407) recently stopped enrollment after meeting protocol-defined criteria for futility, suggesting a low probability of meeting the primary endpoint of demonstrating a significant 30-day VOD/SOSfree survival difference with the sample size estimates and specific methodology used; analyses are ongoing and results are not yet reported, but no safety concerns were described [59]. Overall, there were no significant differences in hemorrhage rates with defibrotide versus controls in published studies, supporting the safety of this therapeutic approach in such critically ill patients $[56,58]$. Early intervention has also been consistently associated with better outcomes in adult and pediatric populations, and published prevention studies show a benefit in high-risk populations with favorable tolerability [58].

GvHD. Based on its mechanism of action, defibrotide is also being studied for GvHD prevention. In a murine model of GvHD, the survival rate was $88 \%$ in the defibrotide-treated group while the untreated group had no survivors; defibrotide benefits were also observed after the occurrence of GvHD [48]. An exploratory analysis of the phase 3 pediatric VOD/SOS prevention trial showed 
that patients who received defibrotide prophylaxis had a lower incidence and severity of acute GvHD versus the control group [58]. A study of defibrotide prophylaxis in adults receiving HCT $(N=195)$ also suggested a role for defibrotide in decreasing incidence and severity of acute GvHD: incidence of acute GvHD was $26 \%$ for patients who received defibrotide pre-HCT, $40 \%$ for those receiving defibrotide post-HCT, and $47 \%$ for those receiving no defibrotide $(P=0.057)$, with a trend toward a lower rate of severe GvHD in the pre-HCT arm versus the other groups $(P=$ 0.051 ) [60]. A phase 2 open-label trial evaluating defibrotide for prevention of acute GvHD after HCT in children and adults completed in May 2020 (NCT03339297) [61].

Defibrotide has demonstrated protective effects on endothelial cells from HCT conditioning and data suggest some efficacy in the prevention of acute GvHD after HCT $[48,61]$. The endothelial cells that cover the vascular tree are directly exposed to damaging factors that occur during the onset and progression of acute GvHD [34]. Previous findings suggested that acute GvHD occurrence is associated with activation and dysfunction of endothelial cells $[34,48]$. These results suggest that the endothelium may be a potential target for GvHD prevention and treatment therapies, of which defibrotide may play an important role in improving endothelial tolerance as part of the GvHD pathobiology.

Transplant-associated thrombotic microangiopathy (TA-TMA). TATMA, another common complication following $\mathrm{HCT}$, is associated with widespread endothelial dysfunction across many organs, providing the rationale for the potential therapeutic use of defibrotide for this condition [62]. In a retrospective study of defibrotide in 17 adults and 22 pediatric patients, TA-TMA resolved in $77 \%$ of patients. Notably, patients with TA-TMA resolution were generally diagnosed earlier than those without TA-TMA resolution, suggesting that earlier identification and defibrotide treatment may improve outcomes [62]. Investigatorinitiated studies for this indication are in progress.

Chimeric antigen receptor T-cell (CAR-T) therapy-associated neurotoxicity. Defibrotide may have potential utility in treating endothelial cell injury associated with cellular therapy, for example, CAR-T therapy-associated neurotoxicity. Patients receiving CAR-T therapy often develop cytokine release syndrome, a systemic inflammatory response, which may require intensive care with respiratory support and can lead to neurotoxicity with symptoms including confusion, hallucinations, and seizure [30, 63]. A study of adults with severe CAR-T therapy-associated neurotoxicity found clinical evidence of endothelial dysfunction, including vascular instability, capillary leak, disseminated intravascular coagulation, and blood-brain barrier disruption, along with elevated biomarkers of endothelial activation and endotheliitis, including vWF, IL-6, and TNF-a [64]. Endothelial stabilizing agents with anti-inflammatory properties, like defibrotide, may help prevent severe neurotoxicity, warranting further study based on defibrotide's demonstrated mechanism of action. An ongoing phase 2 trial is evaluating efficacy and safety of defibrotide for prevention of CAR-T therapy-associated neurotoxicity in patients with relapsed or refractory diffuse large B-cell lymphoma receiving axicabtagene ciloleucel, with preliminary results expected in 2021 (NCT03954106) [65].

Cerebral malaria. Disruption of the blood-brain barrier is known to contribute to manifestations of cerebral malaria, including intracerebral hemorrhage, seizures, and increased intracranial pressure [66]. Multiple mechanisms may underlie blood-brain barrier endothelial cell dysfunction, such as adhesion of parasitized red blood cells to endothelium, inflammatory cytokine response, and blood coagulation, all offering possibilities for therapeutic intervention [66]. A preclinical study using a murine model of cerebral malaria investigated defibrotide effects on aspects of malaria pathogenesis [28]. In vitro, defibrotide blocked tissue factor expression and reduced prothrombinase activity, platelet aggregation, and complement activation, demonstrating its antithrombotic activity along with its ability to be antiinflammatory and reverse endothelial injury. In Plasmodium falciparum culture, adding defibrotide prevented parasite growth, possibly by inhibiting red blood cell invasion by released merozoites [28]. This preclinical evidence suggests that the effects of defibrotide coadministration with anti-malarial drugs may warrant further study.

Sickle-cell disease. Sickle-cell disease is often characterized by painful vaso-occlusive crisis, with underlying mechanisms thought to be multifactorial, involving inflammation, adhesion, and aggregation of sickled red blood cells with endothelial cells and platelets [67]. A preventive treatment targeting these underlying mechanisms could aid in management of the condition and potentially reduce reliance on powerful analgesics and their associated complications [67]. To that end, defibrotide is under investigation in a phase 2 trial (NCT03805581) in patients with sickle-cell disease-associated acute chest syndrome, with results anticipated by 2022 [68].

COVID-19 pneumonia. COVID-19, a disease caused by a new coronavirus, is associated with severe lung complications, such as pneumonia and acute respiratory distress syndrome, but vascular complications predominate, including a unique endotheliitis and associated microangiopathy. Data from murine models of acute lung injury and idiopathic pneumonia syndrome suggest that defibrotide protects against endothelial cell injury and stabilizes endothelial cell integrity in this setting [69]. Researchers are studying the potential role of defibrotide in reducing progression of acute respiratory failure rate in COVID-19 pneumonia, and specifically reversing the endotheliitis that underpins the syndrome and leads to profound endothelial cell dysfunction in advanced disease. Pilot clinical studies and phase 2 trials are underway, with promising early results in both U.S. and E.U. studies [70-73].

\section{CONCLUSIONS}

A large body of both preclinical and clinical literature supports a mechanism of action for defibrotide that reduces endothelial cell activation and damage, thereby restoring the thrombo-fibrinolytic balance and preserving endothelial homeostasis. The totality of evidence suggests that defibrotide exerts endothelial protective effects through downregulating HDACs, with PI3K/AKT and p38 MAPK as the potential intracellular link between defibrotide interaction with the endothelial cell membrane and its downstream effects.

Potential limitations associated with defibrotide treatment include frequent infusions placing a high demand on patients and requiring an inpatient setting, although efforts to produce a subcutaneous formulation are underway. Treatment cost may also impact patient access and utilization; however, results from a Canadian study showed that defibrotide treatment for VOD/SOS with multi-organ dysfunction was cost-effective, with an incremental cost-effectiveness estimate below the current payer threshold [74]. While the clinical utility of defibrotide in treating VOD/SOS has been established, its role in preventing VOD/SOS or treating other diseases that result from endothelial dysfunction, such as GvHD, is under investigation. Ongoing and future clinical studies will provide insights into the therapeutic potential of defibrotide for preventing or treating other conditions driven by endothelial cell activation, endotheliitis, and endothelial dysfunction. In summary, defibrotide is a unique, naturally derived endothelial cell protector that restores thrombo-fibrinolytic balance and preserves endothelial homeostasis through antithrombotic, fibrinolytic, anti-inflammatory, anti-oxidative, and 
anti-adhesion activities. It has proven remarkably safe with broad potential in an array of critical disease states underpinned by endothelial cell damage and inflammation. As such, it constitutes a novel, first-in-class oligonucleotide in a new category of promising biopharmaceuticals, which modulate arguably one of the most important interfaces in human disease, namely the endothelium.

\section{REFERENCES}

1. Richardson PG, Carreras E, lacobelli M, Nejadnik B. The use of defibrotide in blood and marrow transplantation. Blood Adv. 2018;2:1495-509.

2. Defitelio ${ }^{\circledast}$ (defibrotide) [prescribing information]. Palo Alto, CA, USA: Jazz Pharmaceuticals, Inc.; 2016.

3. Pescador R, Capuzzi L, Mantovani M, Fulgenzi A, Ferrero ME. Defibrotide: properties and clinical use of an old/new drug. Vascul Pharmacol. 2013:59:1-10.

4. Palmer KJ, Goa KL. Defibrotide. A review of its pharmacodynamic and pharmacokinetic properties, and therapeutic use in vascular disorders. Drugs. 1993;45:259-94

5. Crystal Research Associates, LLC. Gentium. Drugs for vascular diseases induced by cancer \& cancer treatments. Villa Guardia, Italy: Gentium S.r.l.; 2006. https://cdn2. hubspot.net/hub/150154/file-18094943-pdf/docs/gent_eio_06-26-06.pdf.

6. Richardson PG, Elias AD, Krishnan A, Wheeler C, Nath R, Hoppensteadt D, et al. Treatment of severe veno-occlusive disease with defibrotide: compassionate use results in response without significant toxicity in a high-risk population. Blood. 1998;92:737-44.

7. Richardson PG, Murakami C, Jin Z, Warren D, Momtaz P, Hoppensteadt D, et al. Multi-institutional use of defibrotide in 88 patients after stem cell transplantation with severe veno-occlusive disease and multisystem organ failure: response without significant toxicity in a high-risk population and factors predictive of outcome. Blood. 2002;100:4337-43.

8. Defitelio ${ }^{\circledast}$ (defibrotide) [summary of product characteristics]. Villa Guardia, Italy: Gentium SpA; 2018.

9. European Medicines Agency. Public summary of opinion on orphan designation. Defibrotide for the prevention of graft-versus-host disease. EMA/COMP/627684/ 2013. London, United Kingdom: European Medicines Agency; 2013.

10. Mohty M, Malard F, Abecassis M, Aerts E, Alaskar AS, Aljurf M, et al. Sinusoidal obstruction syndrome/veno-occlusive disease: current situation and perspectives-a position statement from the European Society for Blood and Marrow Transplantation (EBMT). Bone Marrow Transplant. 2015;50:781-9.

11. Fan CQ, Crawford JM. Sinusoidal obstruction syndrome (hepatic veno-occlusive disease). J Clin Exp Hepatol. 2014;4:332-46.

12. Dalle JH, Giralt SA. Hepatic veno-occlusive disease after hematopoietic stem cell transplantation: risk factors and stratification, prophylaxis, and treatment. Biol Blood Marrow Transplant. 2016;22:400-9.

13. Bearman SI. The syndrome of hepatic veno-occlusive disease after marrow transplantation. Blood 1995; 85:3005-20.

14. Carreras E. Early complications after HSCT. In: The EBMT handbook, 6th ed. Cham, Switzerland: European School of Haematology; 2012. p. 177-95.

15. Friedman SL. Molecular regulation of hepatic fibrosis, an integrated cellular response to tissue injury. J Biol Chem. 2000;275:2247-50. https://doi.org/10.1074/ jbc.275.4.2247

16. Coppell JA, Brown SA, Perry DJ. Veno-occlusive disease: cytokines, genetics, and haemostasis. Blood Rev. 2003;17:63-70.

17. Carreras E, Diaz-Ricart M. The role of the endothelium in the short-term complications of hematopoietic SCT. Bone Marrow Transplant. 2011;46:1495-502.

18. Salat C, Holler E, Kolb HJ, Reinhardt B, Pihusch R, Wilmanns W, et al. Plasminogen activator inhibitor-1 confirms the diagnosis of hepatic veno-occlusive disease in patients with hyperbilirubinemia after bone marrow transplantation. Blood. 1997;89:2184-8.

19. Hunt BJ, Jurd KM. Endothelial cell activation. A central pathophysiological process. BMJ. 1998;316:1328-9.

20. Aird WC. Endothelium in health and disease. Pharm Rep. 2008;60:139-43.

21. Bracht F, Schror K. Isolation and identification of aptamers from defibrotide that act as thrombin antagonists in vitro. Biochem Biophys Res Commun. 1994;200:933-7.

22. Mor-Vaknin N, Saha A, Legendre M, Carmona-Rivera C, Amin MA, Rabquer BJ, et al. DEK-targeting DNA aptamers as therapeutics for inflammatory arthritis. Nat Commun. 2017:8:14252.

23. Song W, Ye J, Pan N, Tan C, Herrmann M. Neutrophil extracellular traps tied to rheumatoid arthritis: points to ponder. Front Immunol. 2020;11:578129.

24. Maeda T, Wakasawa T, Shima Y, Tsuboi I, Aizawa S, Tamai I. Prolonged administration of defibrotide is non-immunogenic in rats and dogs. Blood. 2009;114:4180.
25. Umemura K, Iwaki T, Kimura T, Ogawa C, Fukuda T, Taniguchi S, et al. Pharmacokinetics and safety of defibrotide in healthy Japanese subjects. Clin Pharmacol Drug Dev. 2016;5:548-51.

26. Palomo M, Mir E, Rovira M, Escolar G, Carreras E, Diaz-Ricart M. What is going on between defibrotide and endothelial cells? Snapshots reveal the hot spots of their romance. Blood. 2016;127:1719-27.

27. Pasini FL, Frigerio C, Capecchi PL, Ceccatelli L, Messa GL, Franchi M, et al. Modulation of venous endothelial activity and transcellular calcium transport by defibrotide: the adenosine hypothesis. Semin Thromb Hemost. 1996;22:15-20.

28. Francischetti IM, Oliveira CJ, Ostera GR, Yager SB, Debierre-Grockiego F, Carregaro $V$, et al. Defibrotide interferes with several steps of the coagulation-inflammation cycle and exhibits therapeutic potential to treat severe malaria. Arterioscler Thromb Vasc Biol. 2012;32:786-98.

29. Palomo M, Vera M, Martin S, Torramadé-Moix S, Martinez-Sanchez J, Belen Moreno A, et al. Up-regulation of HDACs, a harbinger of uraemic endothelial dysfunction, is prevented by defibrotide. J Cell Mol Med. 2020. 2020;24:1713-23.

30. Lee DW, Gardner R, Porter DL, Louis CU, Ahmed N, Jensen M, et al. Current concepts in the diagnosis and management of cytokine release syndrome. Blood. 2014;124:188-95.

31. Shakespear MR, Halili MA, Irvine KM, Fairlie DP, Sweet MJ. Histone deacetylases as regulators of inflammation and immunity. Trends Immunol. 2011;32:335-43.

32. Zampetaki A, Zeng L, Margariti A, Xiao Q, Li H, Zhang Z, et al. Histone deacetylase 3 is critical in endothelial survival and atherosclerosis development in response to disturbed flow. Circulation. 2010;121:132-42.

33. Palomo M, Diaz-Ricart M, Rovira M, Escolar G, Carreras E. Defibrotide prevents the activation of macrovascular and microvascular endothelia caused by soluble factors released to blood by autologous hematopoietic stem cell transplantation. Biol Blood Marrow Transplant. 2011;17:497-506.

34. Martinez-Sanchez J, Hamelmann H, Palomo M, Mir E, Moreno-Castaño AB, Torramade $S$, et al. Acute graft-vs.-host disease-associated endothelial activation in vitro is prevented by defibrotide. Front Immunol. 2019;10:2339.

35. Koehl GE, Geissler EK, lacobelli M, Frei C, Burger V, Haffner S, et al. Defibrotide: an endothelium protecting and stabilizing drug, has an anti-angiogenic potential in vitro and in vivo. Cancer Biol Ther. 2007;6:686-90.

36. Grimes JM, Grimes KV. p38 MAPK inhibition: a promising therapeutic approach for COVID-19. J Mol Cell Cardiol. 2020;144:63-5.

37. Cella G, Sbarai A, Mazzaro G, Motta G, Carraro P, Andreozzi GM, et al. Tissue factor pathway inhibitor release induced by defibrotide and heparins. Clin Appl Thromb Hemost. 2001;7:225-8.

38. Coccheri S, Biagi G, Legnani C, Bianchini B, Grauso F. Acute effects of defibrotide, an experimental antithrombotic agent, on fibrinolysis and blood prostanoids in man. Eur J Clin Pharmacol. 1988;35:151-6.

39. Echart $\mathrm{CL}$, Graziadio B, Somaini S, Ferro LI, Richardson PG, Fareed J, et al. The fibrinolytic mechanism of defibrotide: effect of defibrotide on plasmin activity. Blood Coagul Fibrinolysis. 2009;20:627-34.

40. Falanga A, Vignoli A, Marchetti M, Barbui T. Defibrotide reduces procoagulant activity and increases fibrinolytic properties of endothelial cells. Leukemia. 2003;17:1636-42.

41. Eissner G, Multhoff G, Gerbitz A, Kirchner S, Bauer S, Haffner S, et al. Fludarabine induces apoptosis, activation, and allogenicity in human endothelial and epithelial cells: protective effect of defibrotide. Blood. 2002;100:334-40.

42. Zhou Q, Chu X, Ruan C. Defibrotide stimulates expression of thrombomodulin in human endothelial cells. Thromb Haemost. 1994;71:507-10.

43. Falanga A, Marchetti M, Vignoli A, Barbui T. Changes in fibrinolysis, coagulation and endothelium activation in patients given defibrotide for hematopoietic stem cell transplant-associated veno-occlusive disease. Thromb Haemost. 1999;Suppl: A529.

44. Coccheri S, Biagi G. Defibrotide. Cardiovasc Drug Rev. 1991;9:172-96.

45. Pellegatta F, Lu Y, Radaelli A, Zocchi MR, Ferrero E, Chierchia S, et al. Druginduced in vitro inhibition of neutrophil-endothelial cell adhesion. $\mathrm{Br} \mathrm{J}$ Pharmacol. 1996;118:471-6.

46. Scalia R, Kochilas L, Campbell B, Lefer AM. Effects of defibrotide on leukocyteendothelial cell interaction in the rat mesenteric vascular bed: role of P-selectin. Meth Find Exp Clin Pharmacol. 1996;18:669-76.

47. Lobel P, Schror K. Selective stimulation of coronary vascular PGI2 but not of platelet thromboxane formation by defibrotide in the platelet perfused heart. Naunyn Schmiedebergs Arch Pharmacol. 1985;331:125-30.

48. García-Bernal $D$, Palomo $M$, Martínez CM, Millán-Rivero JE, García-Guillén Al, Blanquer $M$, et al. Defibrotide inhibits donor leukocyte endothelial interactions and protects against acute graft-versus-host disease. J Cell Mol Med. 2020;24:8031-44.

49. Mitsiades CS, Rouleau C, Echart C, Menon K, Teicher B, Distaso M, et al. Preclinical studies in support of defibrotide for the treatment of multiple myeloma and other neoplasias. Clin Cancer Res. 2009;15:1210-21. 
50. Barash U, Lapidot M, Zohar Y, Loomis C, Moreira A, Feld S, et al. Involvement of heparanase in the pathogenesis of mesothelioma: basic aspects and clinical applications. J Natl Cancer Inst. 2018;110:1102-14.

51. Hallmann R, Horn N, Selg M, Wendler O, Pausch F, Sorokin LM. Expression and function of laminins in the embryonic and mature vasculature. Physiol Rev. 2005;85:979-1000.

52. Ostrovsky O, Shimoni A, Rand A, Vlodavsky I, Nagler A. Genetic variations in the heparanase gene (HPSE) associate with increased risk of GVHD following allogeneic stem cell transplantation: effect of discrepancy between recipients and donors. Blood. 2010;115:2319-28.

53. Benimetskaya L, Wu S, Voskresenskiy AM, Echart C, Zhou JF, Shin J, et al. Angiogenesis alteration by defibrotide: implications for its mechanism of action in severe hepatic veno-occlusive disease. Blood. 2008;112:4343-52.

54. Kaleelrahman M, Eaton JD, Leeming D, Bowyer K, Taberner D, Chang J, et al. Role of plasminogen activator inhibitor-1 (PAI-1) levels in the diagnosis of BMTassociated hepatic veno-occlusive disease and monitoring of subsequent therapy with defibrotide (DF). Hematology. 2003;8:91-95.

55. Richardson PG, Soiffer RJ, Antin JH, Uno H, Jin Z, Kurtzberg J, et al. Defibrotide for the treatment of severe hepatic veno-occlusive disease and multiorgan failure after stem cell transplantation: a multicenter, randomized, dose-finding trial. Biol Blood Marrow Transplant. 2010;16:1005-17.

56. Richardson PG, Riches ML, Kernan NA, Brochstein JA, Mineishi S, Termuhlen AM, et al. Phase 3 trial of defibrotide for the treatment of severe veno-occlusive disease and multi-organ failure. Blood. 2016;127:1656-65.

57. Kernan NA, Grupp S, Smith AR, Arai S, Triplett B, Antin JH, et al. Final results from a defibrotide treatment-IND study for patients with hepatic veno-occlusive disease/sinusoidal obstruction syndrome. Br J Haematol. 2018;181:816-27.

58. Corbacioglu S, Cesaro S, Faraci M, Valteau-Couanet D, Gruhn B, Rovelli A, et al. Defibrotide for prophylaxis of hepatic veno-occlusive disease in paediatric haemopoietic stem-cell transplantation: an open-label, phase 3, randomised controlled trial. Lancet. 2012;379:1301-9.

59. Jazz Pharmaceuticals. Jazz Pharmaceuticals stops enrollment in phase 3 study evaluating defibrotide for the prevention of veno-occlusive disease [press release]. New York, NY: PR Newswire Association LLC; 2020.

60. Tekgündüz E, Kaya AH, Bozdağ SC, Koçubaba Ş, Kayıkçı Ö, Namdaroğlu S, et al. Does defibrotide prophylaxis decrease the risk of acute graft versus host disease following allogeneic hematopoietic cell transplantation? Transfus Apher Sci. 2016;54:30-34.

61. ClinicalTrials.gov. An open-label study of defibrotide for the prevention of acute graft-versus-host-disease (AGvHD). 2020.

62. Yeates L, Slatter MA, Bonanomi S, Lim F, Ong SY, Dalissier A, et al. Use of defibrotide to treat transplant-associated thrombotic microangiopathy: a retrospective study of the Paediatric Diseases and Inborn Errors Working Parties of the European Society of Blood and Marrow Transplantation. Bone Marrow Transplant. 2017;52:762-4.

63. Maude SL, Frey N, Shaw PA, Aplenc R, Barrett DM, Bunin NJ, et al. Chimeric antigen receptor $\mathrm{T}$ cells for sustained remissions in leukemia. $\mathrm{N}$ Engl J Med. 2014;371:1507-17.

64. Gust J, Hay KA, Hanafi LA, Li D, Myerson D, Gonzalez-Cuyar LF, et al. Endothelial activation and blood-brain barrier disruption in neurotoxicity after adoptive immunotherapy with CD19 CAR-T Cells. Cancer Discov. 2017;7:1404-19.

65. ClinicalTrials.gov. A safety and efficacy study of defibrotide in the prevention of chimeric antigen receptor-t-cell-associated neurotoxicity; 2020.

66. Nishanth G, Schluter D. Blood-brain barrier in cerebral malaria: pathogenesis and therapeutic intervention. Trends Parasitol. 2019;35:516-28.

67. Darbari DS, Sheehan VA, Ballas SK. The vaso-occlusive pain crisis in sickle cell disease: Definition, pathophysiology, and management. Eur J Haematol. 2020;105:237-46.

68. ClinicalTrials.gov. Defibrotide in sickle cell disease-related acute chest syndrome; 2020.

69. Klein OR, Choi SW, Haile A, Ktena YP, Pierce E, Smith M, et al. Defibrotide modulates pulmonary endothelial cell activation and protects against lung inflammation in pre-clinical models of LPS-induced lung injury and idiopathic pneumonia syndrome. Biol Blood Marrow Transplant. 2020;6:S138-9.

70. ClinicalTrials.gov. Defibrotide in COVID-19 pneumonia (DEFI-VID19); 2020.

71. Michigan Institute for Clinical \& Health Research. MICHR helps launch novel COVID-19 clinical trial. Ann Arbor, Michigan: MICHR; 2020.

72. Richardson E, Carlo-Stella C, Jara R, Vlodavsky I, lacobelli M, Fareed J et al. Response to Maccio et al., "Multifactorial pathogenesis of COVID-19-related coagulopathy: can defibrotide have a role in the early phases of coagulation disorders?". J Thromb Haemost. 2020. https://doi.org/10.1111/jth.15088
73. EBMT. Haematological therapies at the crossroads of the COVID-19 pandemic. Special session presented at: EBMT 2020 Annual Meeting; September 1, 2020; Madrid, Spain

74. Belsey J, Kemadjou EN, Isaila M, Villa KF. Cost-effectiveness of defibrotide for the treatment of veno-occlusive disease/sinusoidal obstruction syndrome (VOD/SOS) with multi-organ dysfunction (MOD) post-hematopoietic Stem cell transplantation (HSCT) in Canada. Blood. 2018;132 Suppl 1:4702.

\section{ACKNOWLEDGEMENTS}

Medical writing and editorial assistance were provided by Michelle Hughes, PhD, and Nancy Tang, PharmD, of Cello Health Communications/SciFluent Communications, Inc., and were financially supported by Jazz Pharmaceuticals.

\section{AUTHOR CONTRIBUTIONS}

All authors contributed to the initial concept and design of this review manuscript, critically revised the drafts, approved the final version, and agree to be accountable for all aspects of the work in ensuring that questions related to the accuracy or integrity of any part of the work are appropriately investigated and resolved.

\section{COMPETING INTERESTS}

PGR has served on advisory committees and has received research funding from Jazz Pharmaceuticals. MP has received honoraria in speaker's fee for a symposium from Jazz Pharmaceuticals. NAK received grants from Gentium during the conduct of the study, and her research was supported by the National Cancer Institute of the National Institutes of Health under award number P30 CA008748; the content is solely the responsibility of the author and does not necessarily represent the official views of the National Institutes of Health. GCH has stocks/ownership interests in Sangamo Bioscience, Axim Biotechnologies, Juno Therapeutics, Kite Pharma, Novartis, Insys Therapeutics, AbbVie, GW Pharmaceuticals, Cardinal Health, Immunomedics, Endocyte, Clovis Oncology, Cellectis, Aetna, CVS Health, Celgene, Bluebird Bio, Bristol Myers Squibb/Medarex, CRISPR Therapeutics, IDEXX Laboratories, Johnson \& Johnson, Pfizer, Procter \& Gamble, Vertex, Bayer, and Scotts-Miracle; has served in advisory/consulting roles for Pfizer, Kite Pharma, Incyte, and Jazz Pharmaceuticals; has received research funding from Takeda, Jazz Pharmaceuticals, Astellas, Incyte, and Pharmacyclics; and has received travel, accommodations, and/or expense reimbursement from Kite Pharma, Incyte, Pfizer, Falk Foundation, Jazz Pharmaceuticals, and Astellas Pharma. NC has nothing to disclose. EC has received research funds and honoraria in speaker's fees for symposia from Jazz Pharmaceuticals/ Gentium Inc.

\section{ADDITIONAL INFORMATION}

Correspondence and requests for materials should be addressed to Paul G. Richardson.

Reprints and permission information is available at http://www.nature.com/reprints

Publisher's note Springer Nature remains neutral with regard to jurisdictional claims in published maps and institutional affiliations.

\begin{abstract}
Attribution 4.0 International License, which permits use, sharing, adaptation, distribution and reproduction in any medium or format, as long as you give appropriate credit to the original author(s) and the source, provide a link to the Creative Commons license, and indicate if changes were made. The images or other third party material in this article are included in the article's Creative Commons license, unless indicated otherwise in a credit line to the material. If material is not included in the article's Creative Commons license and your intended use is not permitted by statutory regulation or exceeds the permitted use, you will need to obtain permission directly from the copyright holder. To view a copy of this license, visit http://creativecommons. org/licenses/by/4.0/.
\end{abstract}

Open Access This article is licensed under a Creative Commons

(c) The Author(s) 2021 